\section{REGULATION OF THE AORTIC VALVE OPENING}

\author{
In vivo dynamic \\ measurement of aortic \\ valve orifice area
}

Aortic valve orifice area was dynamically measured in anesthetized dogs with a new measuring system involving electromagnetic induction. This system permits us real-time measurement of the valve orifice area in beating hearts in situ. The aortic valve was already open before aortic pressure started to increase without detectable antegrade aortic flow. Maximum opening area was achieved while flow was still accelerating at a mean of 20 to 35 msec before peak blood flow. Maximum opening area was affected by not only aortic blood flow but also aortic pressure, which produced aortic root expansion. The aortic valve orifice area's decreasing curve (corresponding to valve closure) was composed of two phases: the initial decrease and late decrease. The initial decrease in aortic valve orifice area was slower $\left(4.1 \mathrm{~cm}^{2} / \mathrm{sec}\right)$ than the late decrease $\left(28.5 \mathrm{~cm}^{2} / \mathrm{sec}\right)$. Aortic valve orifice area was reduced from maximum to $40 \%$ of maximum (in a triangular open position) during the initial slow closing. These measurements showed that (1) initial slow closure of the aortic valve is evoked by leaflet tension which is produced by the aortic root expansion (the leaflet tension tended to make the shape of the aortic orifice triangular) and (2) late rapid closure is induced by backflow of blood into the sinus of Valsalva. Thus, cusp expansion owing to intraaortic pressure plays an important role in the opening and closing of the aortic valve and aortic blood flow. (J Thorac Cardiovasc Surg 1995;110:496-503)

Masafumi Higashidate, MD, ${ }^{a}$ Kouichi Tamiya, MD, ${ }^{\mathrm{b}}$ Toshiyuki Beppu, MS, ${ }^{\mathrm{b}}$ and Yasuharu Imai, MD, ${ }^{\mathbf{a}}$ Tokyo, Japan
$A_{\mathrm{in}}^{\mathrm{n}}$ estimation of orifice area of the aortic valve is important for the proper evaluation of patients who are potential candidates for aortic valve operation. Until recently most of the studies describing the behavior of the normal aortic valve were carried out either with models or at autopsy. ${ }^{1,2}$ However, the valve movements observed in these studies in nonbeating hearts may not be representative of aortic valve behavior in the actively contracting heart in vivo. Observations of aortic valve action in the beating heart has been done both indirectly and directly. Indirect methods include $\mathrm{x}$-ray technique, with either contrast material ${ }^{3}$ or radiopaque mark-

From the Department of Pediatric Cardiovascular Surgery and Surgical Science, ${ }^{\mathrm{b}}$ The Heart Institute of Japan, Tokyo Women's Medical College, Tokyo, Japan.

Received for publication Dec. 22, 1992.

Accepted for publication Dec. 22, 1994.

Address for reprints: Masafumi Higashidate, MD, Department of Pediatric Cardiovascular Surgery, The Heart Institute of Japan, Tokyo Women's Medical College, Kawada-cho 8-1, Shinjuku-ku, Tokyo 162, Japan.

Copyright @ 1995 by Mosby-Year Book, Inc.

$0022-5223 / 95 \$ 3.00+0 \quad \mathbf{1 2 / 1 / 6 3 2 9 1}$ ers, ${ }^{4}$ as well as echocardiography. ${ }^{5}$ A major disadvantage of these methods is either the low time resolution or the observation of only two of the three leaflets. Direct observation of aortic valve action in the intact animal can be achieved with cinematographic techniques. ${ }^{6}$ The main problem with this method is the replacement of blood by transparent medium for a period time sufficiently long to allow observation and photographic recording of the valve. The duration of direct recording of the aortic valve cusps under physiologic conditions is limited because of the small oxygen content of the hemoglobin-free perfusion fluid. In addition, these recordings were not real-time, and the full scope of the aortic valve was not monitored.

In the present study, orifice area of the aortic valve was measured in open-chest dogs with a real-time area measuring system. ${ }^{7}$ This new measuring system used lock-in amplification to pick up the electric signal with an one-turn loop stitched along the free edges of the aortic valve cusps in open-chest anesthetized dogs. A constant magnitude-alternating electromagnetic field was supplied from the outside of the body and induced the electrical 
signals. Induced electrically evoked potentials at both ends of the one-turn loop were proportional to the projection area of valve orifice area. This system permitted us to measure orifice area in beating hearts in vivo without any constraints to the valve motion or serious disturbances in the animal blood circulation. ${ }^{7-10}$

\section{Material and methods}

Experimental procedure. Six mongrel dogs weighing from 12 to $18 \mathrm{~kg}$ were anesthetized with pentobarbital ( 25 $\mathrm{mg} / \mathrm{kg}$ ), the chest was opened at the midsternum, and the heart was exposed. The dogs were intubated with a cuffed endotracheal tube, and their lungs were ventilated with a Bird respirator (model MK-8; Bird Corp., Palm Springs, Calif.). The inferior and superior venae cavae and the ascending aorta were cannulated for the cardiopulmonary bypass with a pulsatile pump (PSA-120; Tonokura Ika Kogyo Co. Ltd., Tokyo, Japan). An aortotomy was performed just above the aortic sinuses, and a metal thread which functions as a sensing loop of the electromagnetic field was stitched along the free edges of the aortic valve cusps with gathered stitches. The metal thread consisted of three fine urethane resin-coated copper wires $(25 \mu \mathrm{m}$ diameter; Sumitomo Denko, Osaka, Japan). Both ends of the metal thread were guided out from the aorta through a single pinhole in the aortic walls. After visual confirmation of the appropriate arrangement of the sensing loop along the valve cusps in the aorta, the incision in the aorta was sutured and original blood circulation was reestablished. A drive coil assembly was placed perpendicular to the extension of the long axis of the heart and was directed toward the aortic valve region. Left ventricular and aortic pressures were measured with high-fidelity cathetertipped transducers (Micro-Tip model PC-350; Millar Instruments Inc., Houston, Tex.). The left ventricular catheter was inserted through an apical puncture, and access to the aorta was gained by retrograde passage via the right common carotid artery. The aortic pressure sensor was positioned just above the aortotomy. Aortic flow (cardiac output) was continuously measured just above aortic sinuses that correspond to aortotomy suture with an ultrasonic flow probe (Transonic T101; Transonic System Inc., Ithaca, N.Y.).

All dogs received humane care in compliance with the "Principles of Laboratory Animal Care" formulated by the National Society for Medical Research and the "Guide for the Care and Use of Laboratory Animals" prepared by the Institute of Laboratory Animal Resources and published by the National Institutes of Health (NIH Publication No. 86-23, revised 1985).

Measuring system. The real-time area measuring system used in this study has been described in detail in our previous reports ${ }^{7-10}$ and will be discussed here only briefly. This equipment for monitoring the aortic valve orifice area (AVOA) is based on electromagnetic induction. Three alternating carrier signals $(96,100$, and $104 \mathrm{kHz})$ of constant magnitude were supplied from three drive coils assembled on a board base. The axes of the three coils, which were directed toward the aortic valve, intersect with each other at vertically opposite angles of 20 degrees apart, $1 \mathrm{~m}$ from the aortic valve region. The aortic valve orifice consists of three free edges of the aortic cusps and is encircled with gathered stitches with a piece of extremely fine pliable metal thread that consists of three urethane resin-coated copper wires ( $25 \mu \mathrm{m}$ diameter). This one-turn loop of the sensing wire, which works as a receiving antenna for the three carrier signals, is connected to a preamplifier made of a high-frequency operational amplifier with a short segment of fine shielded wire. The magnitude of the alternating electric potentials ( 5 to $10 \mathrm{mV}$ ) containing the three signals induced in a sensing loop is proportional to the area enclosed by the constant electromagnetic field; this area is predominantly that of the AVOA. Returning the sensing loop to the rigid metal triangle $\left(1.73 \mathrm{~cm}^{2}\right)$ positioned at the aortic valve region adjacent to the heart provides the standard signal for calibration of the system.

Calculations. Fig. 1 is a schematic representation of the normal relations between aortic pressure $(\mathrm{AoP})$, aortic flow, and AVOA. Stroke volume was calculated with planimetry of the area under the calibrated aortic flow curve and cardiac output (mean flow) was recorded with the flowmeter. Maximum valve orifice area (S1 in Fig. 1) was measured as the highest point of the AVOA, and the onset of rapid closure is named as $\mathrm{S} 2$ in Fig. 1. Aortic flow occurred during the time between point $\mathrm{O}$ and $\mathrm{C}$ (corresponding to dicrotic notch) on the AoP recording. Peak flow was measured as the highest point of the aortic flow curve (F1). The rate of changes in AVOA curves were as follows: the S0-S1 slope gave the rate of valve opening, the S1-S2 slope gave the rate of initial slow closing, and the S2-Sc slope gave the speed of the rapid valve closure. Aortic valve opening time was defined between point $O$ and point $\mathrm{C}$ (dicrotic notch) on AoP recording. At the end of experiment, all dogs were killed, and the cross-sectional areas of aortic root at the level of commissura were measured with in round slice sectioning.

\section{Results}

Because the aortic valve opens and closes rapidly (16.9 and $25.4 \mathrm{msec}$ ), it was impossible to perform continuous recordings of the changes in valve orifice during cardiac cycles. However, we measured it continuously with a new measuring system involving electromagnetic induction.

Fig. 1 is a original record taken from a dog experiment showing typical relations among aortic flow, pressure, and AVOA. A regular heart rhythm and normal aortic flow, as well as physiologic aortic and left ventricular pressures were maintained during all the experiments. Table I summarizes the hemodynamic and AVOA data. Table II summarizes time intervals between the position on pressure and flow and onsets of AVOA changes in the seven dogs examined. In each experiment, measurements were taken under stable conditions on ten consecu- 

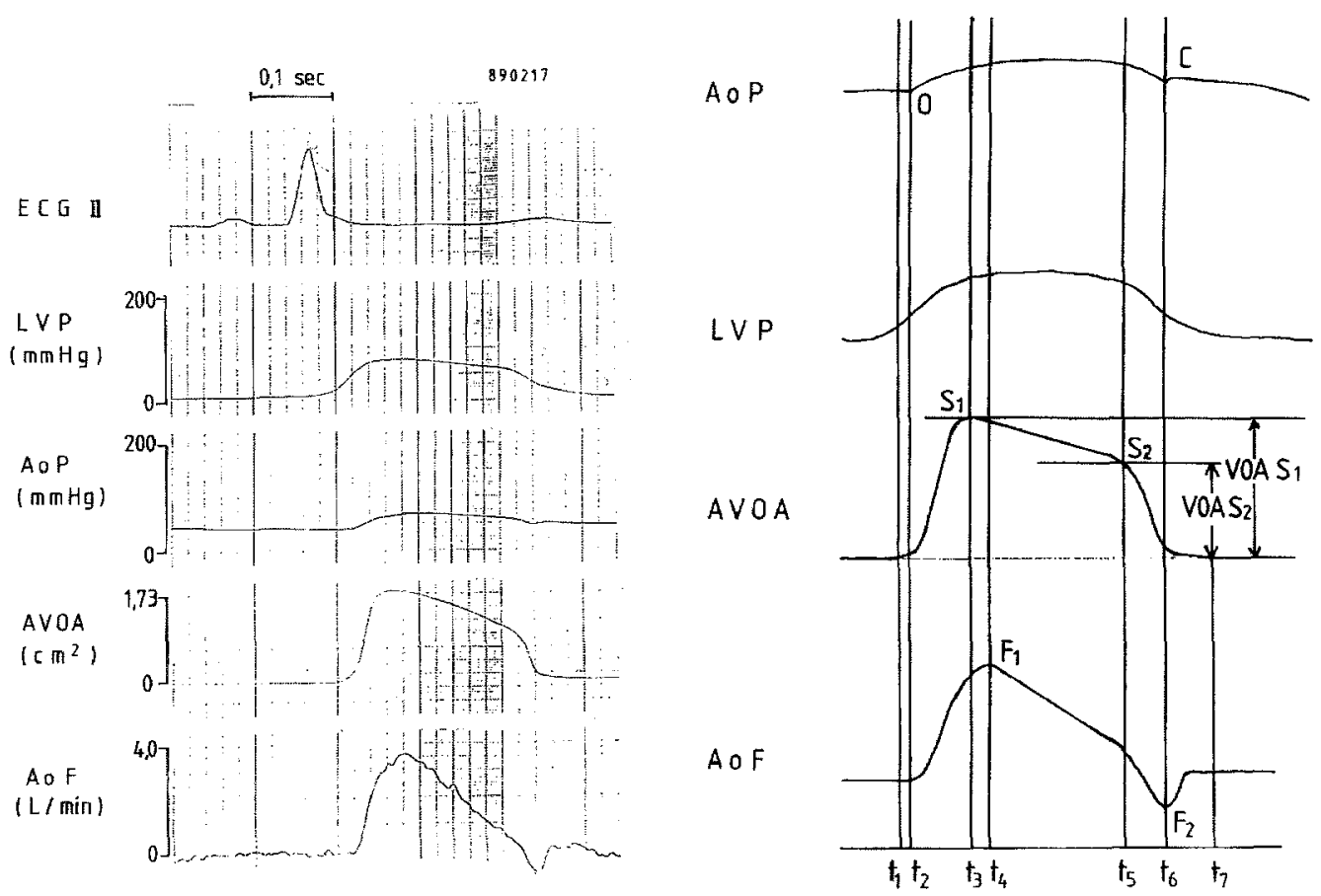

Fig. 1. Left panel, Relations between hemodynamic data and AVOA. $O$, Onset of ascending aortic pressure elevation; $C$, dicrotic notch; $L V P$, left ventricular pressure; $S 1$, peak point of AVOA; $S 2$, inflection point of the decreasing curve of AVOA; $A o F$, aortic blood flow; $F 1$, peak of forward flow; $F 2$, peak of back flow. $t 1-t 2$, initial slow opening; $t 2-t 3$, rapid opening; $t 3-t 4$, time delay between maximum AVOA and peak aortic flow; $t 3-t 5$, initial slow closing; $t 5-t 6$, late rapid closing; $t 6-t 7$, complete closing. Right panel, An original record showing the relation between left ventricular pressure, AoP, AVOA, and aortic blood flow. Before aortic pressure elevation and aortic flow started, AVOA was already increased. Note that AVOA has already begun to rise before the onset of AoP elevation and aortic flow.

Table I. Hemodynamic and dimensional data from seven dogs

\begin{tabular}{|c|c|c|c|c|c|c|c|c|}
\hline Dog No. & $\begin{array}{c}\text { Body } \\
\text { weight } \\
(\mathrm{kg})\end{array}$ & $\begin{array}{c}C O \\
(L / m i n)\end{array}$ & $\begin{array}{c}H R \\
(m i n)\end{array}$ & $\begin{array}{c}\text { AoP systolic/ } \\
\text { diastolic } \\
\text { (mm Hg) }\end{array}$ & $\begin{array}{c}\text { Stroke } \\
\text { volume } \\
(m l)\end{array}$ & $\begin{array}{c}\text { Aortic root } \\
\text { area } \\
\left(\mathrm{cm}^{2}\right)\end{array}$ & $\begin{array}{c}S 1 \\
\left(\mathrm{~cm}^{2}\right)\end{array}$ & $\begin{array}{c}S 2 \\
\left(\mathrm{~cm}^{2}\right)\end{array}$ \\
\hline 1 & 13 & 0.53 & 158 & $100 / 70$ & 3.5 & 0.64 & 1.04 & 0.52 \\
\hline 2 & 16 & 0.85 & 142 & $150 / 100$ & 6.0 & 0.78 & 1.56 & 0.77 \\
\hline 3 & 15 & 0.70 & 150 & $140 / 110$ & 4.7 & 0.64 & 1.51 & 0.77 \\
\hline 4 & 10 & 0.76 & 115 & $95 / 80$ & 6.6 & 0.60 & 1.50 & 0.70 \\
\hline 5 & 10 & 0.70 & 111 & $100 / 75$ & 6.3 & 0.50 & 1.70 & 1.10 \\
\hline 6 & 9 & 0.65 & 160 & $95 / 75$ & 3.8 & 0.38 & 0.95 & 0.52 \\
\hline 7 & 18 & 0.94 & 118 & $120 / 98$ & 7.6 & 0.94 & 1.94 & 1.08 \\
\hline Mean & 13 & 0.71 & 131 & $105.7 / 79.7$ & 5.50 & 0.64 & 1.46 & 0.78 \\
\hline Standard deviation & 3.2 & 0.11 & 22.1 & $16.6 / 18.0$ & 1.53 & 0.17 & 0.36 & 0.24 \\
\hline
\end{tabular}

$C O$, Cardiac output; $H R$, heart rate; $S 1$, aortic valve orifice area at point $S 1$ in Fig. 1; $S 2$, aortic valve orifice area at point $S 2$ in Fig. 1.

tive beats; the means \pm standard deviation are given.

In Fig. 1, the aortic valve was already open about $8 \%$ of its maximum before aortic pressure started to increase (point $\mathrm{X}$ in Fig. 1). The time duration between the aortic valve opening and the onset of the aortic pressure increasing was $20.6 \pm 3.2 \mathrm{msec}$
(Table II, T1-T2). The present data showed that the minimal initial opening of the aortic valve occurs without any detectable forward flow and in the absence of an elevation of aortic pressure.

The rapid opening of the aortic valve occurred simultaneously with the onset of aortic flow, which also coincides with the fact that left ventricular 
pressure exceeded aortic pressure (Fig. 1, Table II). The rapid opening of the valve was smooth, with an average speed of $34.2 \mathrm{~cm} / \mathrm{sec}$. Maximum opening was achieved while flow was still accelerating at $16.9 \pm 5.4 \mathrm{msec}$ (mean \pm standard deviation) before peak flow. The onset of maximum valve opening always precedes the onset of peak aortic flow (16.9 msec; $\mathrm{t} 3-\mathrm{t} 4 \mathrm{in}$ Table II). The duration of aortic valve opening lasts $36.2 \pm 8.9$ msec (mean \pm standard deviation). Just after AVOA attained its maximum, AVOA started decreasing slowly toward valve closure. The initial decrease in AVOA (S1-S2 slope in Fig. 1) is slow $\left(4.1 \mathrm{~cm}^{2} / \mathrm{sec}\right)$, and the late decrease in AVOA (S2-Sc in Fig. 1) was faster $\left(28.3 \mathrm{~cm}^{2} / \mathrm{sec}\right)$. It was more apparent in beats with large stroke volume and was a constant phenomenon persisting in every beat, although the slope of AVOA changed different degrees. The correlation between AVOA at onset of rapid decrease (S2 in Fig. 1) and aortic pressure at that time is shown in Fig. 2 in one dog. This graph has the positive correlation $(r=0.97)$ of S2 with aortic pressure (Fig. 2).

Final closure of the valve was achieved just after the maximum back flow (Fig. 1). The mean speed of AVOA decrease during late rapid closure was 28.3 $\mathrm{cm}^{2} / \mathrm{sec}$. Although stroke volume varied from beat to beat, it was shown that with larger stroke volume the rate of aortic valve opening was faster, hence the maximum AVOA occurred sooner.

Effects of volume loading. To test the effects of volume loading on AVOA, we carried out a rapid intravenous blood transfusion via the venous line. Volume loading produced elevations of AoP, left ventricular pressure, aortic blood flow (AoF), and an increase in maximum AVOA. The correlation between AoP, AoF, and AVOA is shown in the original record taken from one dog; we plotted a graph of the AoF and maximum AVOA (Fig. 3). Maximum AVOA increases with the aortic flow; however, the relationship of both is nonlinear correlation (Fig. 3).

Effects of inflow occlusion. During a brief inflow occlusion which was caused by two snares around superior and inferior venae cavae ligation, aortic flow was decreased, left ventricular pressure was decreased, AoP was decreased, and maximum AVOA was decreased (Fig. 4). We plotted a graph of the AoF and maximum AVOA shown in Fig. 4, and it is a nonlinear correlation (Fig. 4).

Effects of ascending aorta constriction. During ascending aorta constriction (ligation of the ascending at aorta about $2 \mathrm{~cm}$ above aortotomy suture),
Table II. Time intervals between pressure and the onset of $A V O A$ changes

\begin{tabular}{lcccccc}
\hline Dog No. & $\begin{array}{r}t 1-t 2 \\
(m s e c)\end{array}$ & $\begin{array}{r}t 2-t 3 \\
(m s e c)\end{array}$ & $\begin{array}{r}t 3-t 4 \\
(m s e c)\end{array}$ & $\begin{array}{r}t 3-t 5 \\
(m s e c)\end{array}$ & $\begin{array}{r}t 5-t 6 \\
(m s e c)\end{array}$ & $\begin{array}{r}t 6-t 7 \\
(m s e c)\end{array}$ \\
\hline 1 & 20 & 30 & 20 & 75 & 30 & 32 \\
2 & 18 & 25 & 10 & 124 & 20 & 40 \\
3 & 18 & 40 & 14 & 84 & 28 & 20 \\
4 & 20 & 38 & 18 & 144 & 20 & 32 \\
5 & 24 & 56 & 20 & 148 & 32 & 38 \\
6 & 18 & 28 & 10 & 78 & 20 & 20 \\
7 & 26 & 32 & 26 & 108 & 24 & 36 \\
Mean & 20.6 & 36.2 & 16.9 & 106.7 & 25.4 & 31.1 \\
Standard & 3.2 & 8.9 & 5.4 & 27.9 & 4.3 & 8.2 \\
$\quad$ deviation & & & & & & \\
\hline
\end{tabular}

$\mathrm{t} 1, \mathrm{t} 2, \mathrm{t} 3, \mathrm{t} 4, \mathrm{t}, \mathrm{t}, \mathrm{t}, \mathrm{t}$ are included in Fig. $1 . t 1-t 2$, Initial slow opening; $t 2-t 3$, rapid opening; $t 3-t 4$, time difference between maximum AVOA and peak aortic flow; $t 3-t 5$, initial slow closing; $t 5-t 6$, late rapid closing; $t 6-t 7$, complete closing.

ascending AoP elevated, left ventricular pressure elevated, stroke volume decreased, and maximum AVOA was increased (Fig. 5). Compared with volume loading (Fig. 3), aortic constriction induced reduction in $\mathrm{AoF}$, but maximum AVOA increased. Conversely, inflow occlusion induced reduction in AoF, and maximum AVOA decreased (Fig. 4).

\section{Discussion}

In our previous work, we reported the phasic changes in tricuspid valve orifice area using the "areameter" that involved the use of electromagnetic induction. This system permitted real-time, accurate monitoring of the area enclosed by the edges of valve leaflets in situ without artificial constraint to the valve motion. However, the metal threads ( $25 \mu \mathrm{m}$ in diameter) with which gathering stitches were placed on the edges of the three cusps tended to stay in position because they are more rigid than the free edges of the cusps without stitches. In addition, coaptation of the cusps occurs such that they create a coaptation zone. Thus, neither zero AVOA nor nonzero AVOA may afford an accurate prediction of the cusp edge approximation. Actually, the area signal recorded in this method reflects the total AVOA. Although the accuracy of the present technique is circumscribed, the values of AVOA with this technique is reliable and it does not disturb valve movements.

Our finding that aortic valve opens before the onset of aortic blood flow (Fig. 1) is consistent with the finding of Thubrikar, Bosher, and Nolan ${ }^{11}$ that no flow or pressure gradient across the valve is needed to open the valve initially. These observa- 


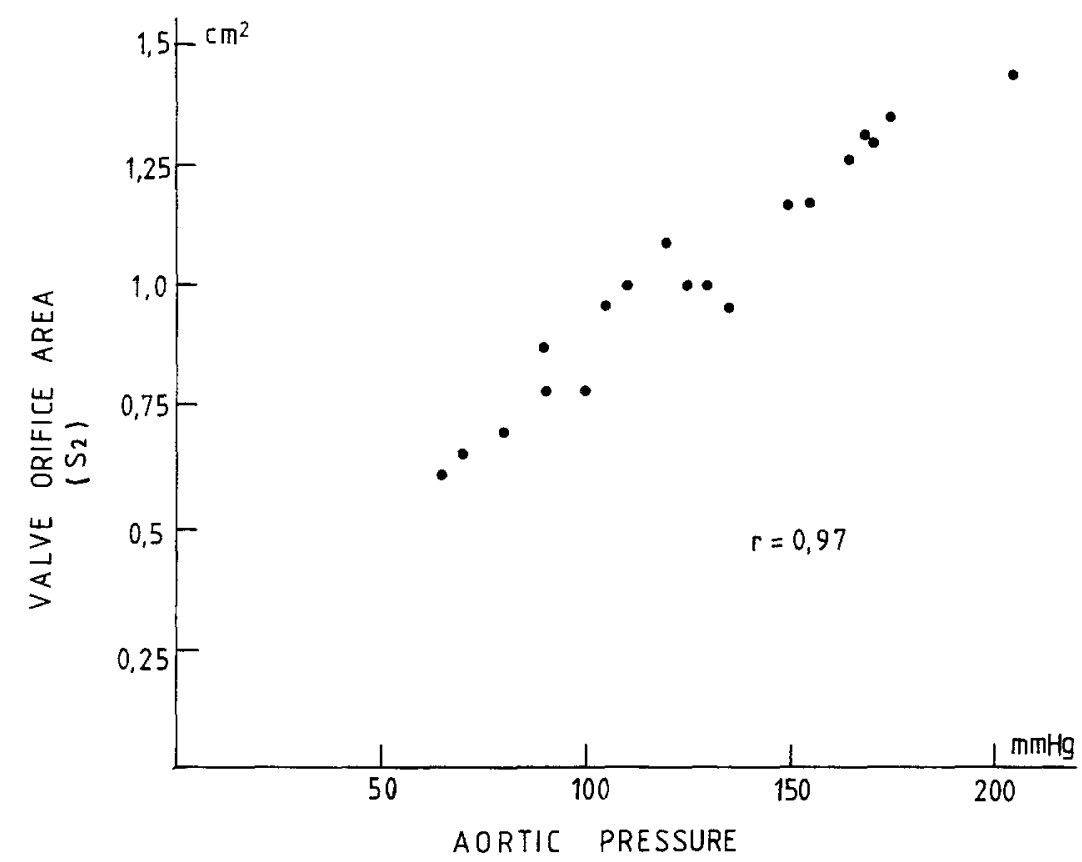

Fig. 2. Relationship of inflection point of the decreasing curve of AVOA (S2) and AoP. AVOA (S2) was measured at the onset of rapid late closing. AoP was also measured with peak systolic pressure. This graph has the positive correlation $(r=0.97)$ of $\mathrm{S} 2$ with AoP, suggesting that $\mathrm{S} 2$ is a triangular open point.

tions suggest that the aortic valve opens before the onset of aortic blood flow because of the increase in the restoring force that tends to make a valve orifice triangular, owing to the increase in the distances between the commissures without forward flow. The reason for this is inferred from the fact that the left ventricular pressure elevation induces aortic root expansion, subsequent commissure (leaflet attachment) separation, and stretching of the free edges of elastic valve leaflet. Therefore, our data support the concept of Thubrikar, Bosher, and Nolan ${ }^{11-13}$ that the valve has an intrinsic potential to open. Our data did not corroborate the hypothesis of Van Steenhoven and colleagues ${ }^{6}$ that the valve opened coincidently with the beginning of the aortic flow. ${ }^{6}$

The second phase, rapid aortic valve opening phase, was fast and was completed within $36.2 \pm 8.9$ msec (Table II). Aortic flow, at this point, then reached a value of about $75 \%$ of its maximum value (Fig. 1). The duration of the rapid opening phase (t2-t3, in Fig. 1) (36.2 \pm 8.9 msec) found in our study is in accordance with that described (a mean of 32 msec) by Van Steenhoven and colleagues ${ }^{6}$ and somewhat longer than that described by Laniado and colleagues ${ }^{5}(26.6 \pm 0.8 \mathrm{msec})$. Conversely, the valve opening time obtained in our study might have been biased to be longer because of low cardiac output caused by anesthesia, artificial ventilation, and surgical procedures such as open chest operations.

At the instant that the ventricular pressure exceeds the aortic pressure, flow starts and the valve opens rapidly, achieving its maximum opening $16.9 \pm 5.4 \mathrm{msec}$ before peak flow, and starts its slow movement toward closure while flow is still accelerating (Fig. 1, Table II). Laniado and colleagues ${ }^{5}$ also showed with echocardiography that complete valve opening preceded peak aortic flow by an average of $43 \mathrm{msec}$ and the cusps started to move toward a closure while flow was still accelerating. It appears that internal forces stored by the displacement of the cusps, in combination with vortexes in the sinuses, cause the leaflets to begin to close before the onset of flow deceleration. It suggests that the aortic valve can close completely without regurgitation in conjunction with elastic valve cusps which produce internal forces by expansion valve cusps. ${ }^{11,13,14}$

Point $\mathrm{S} 2$ in Fig. 1 is defined as a point of inflection in the decreasing curve of the AVOA, which concurs fairly well with Thubrikar's triangular open point. ${ }^{11}$ In the time and area relationship observed with direct cinematographic recordings of aortic valve motion, ${ }^{6}$ this point obviously coincides with the tri- 

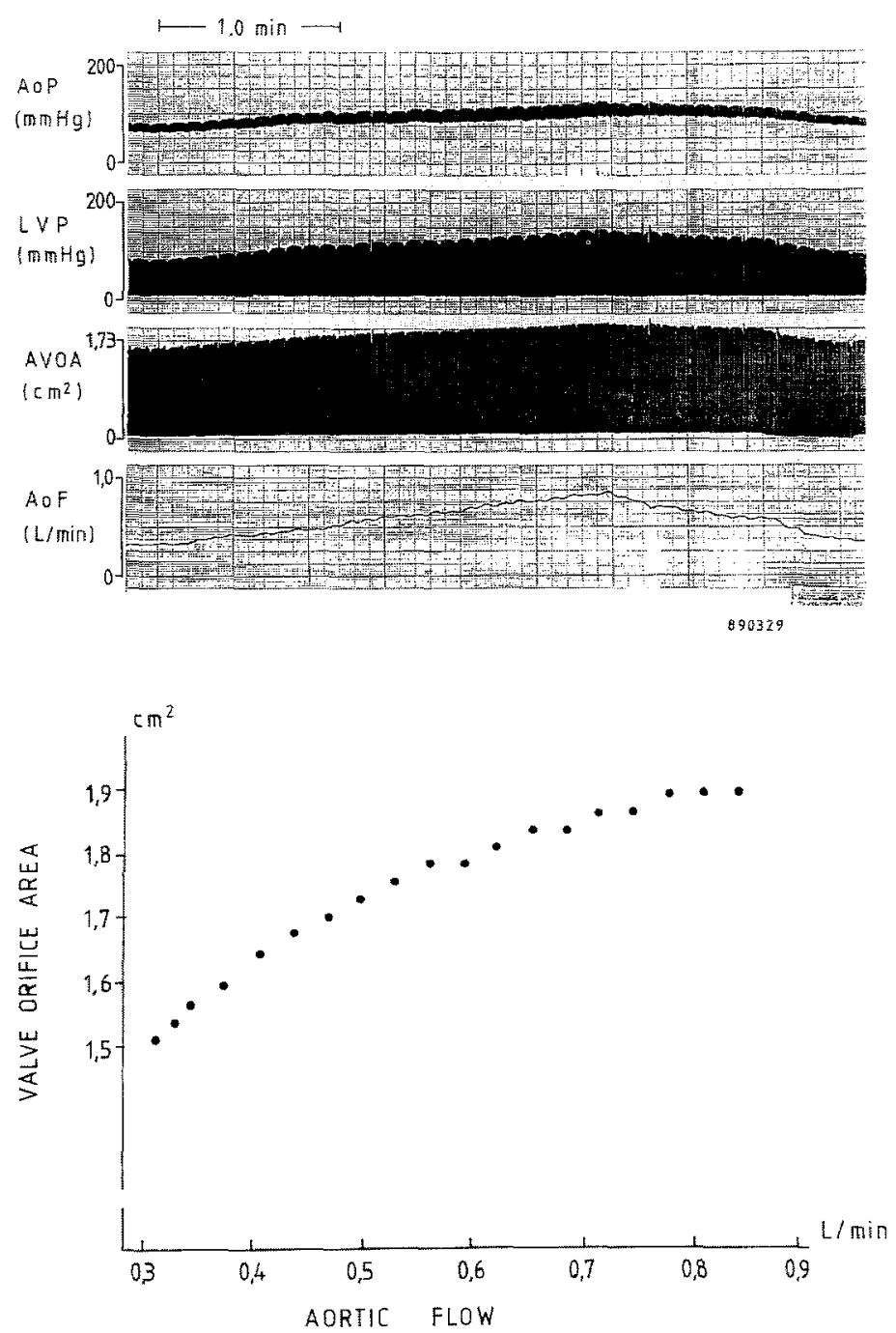

Fig. 3. Upper panel, Effects of rapid blood transfusion on AVOA. LVP, Left ventricular pressure; $A o F$, aortic blood flow. Maximum AVOA increased with AoF increasing. Lower panel, Relationship between maximum AVOA and AoF at blood transfusion (volume loading). Maximum AVOA increased with increasing of the aortic blood flow; however, the relationship of both is a nonlinear correlation.

angular open point. This triangular area, which is calculated as $41 \%$ of its maximum circular open, agrees with our observations area $40 \%$ of maximum AVOA (Table I). This area is calculated as follows: The inner circle area of the aorta is calculated $\left(\pi \mathrm{r}^{2}\right.$; radius $=\mathbf{r}$ ) and a triangle area which is inscribed in the circle is also calculated $\left(3 \sqrt{3 \cdot r^{2}} / 4\right)(41 \%$ of the circle area). When AVOA is reduced to this triangular point (AVOA at point S2 in Fig. 1), backflow may easily induce the eddies in the sinus of Valsalva to produce complete valve closure. We believe that this mechanism plays an important role in closing the valve initially.

S2 areas were gradually increased with aortic pressure during volume loading and aortic constriction (Figs. 2 and 5). Strong correlation was found to exist between the S2 area and aortic pressure (Fig. 2). The correlation coefficient is 0.97 . These data indicate that aortic root expansion caused by aortic pressure elevation increases S2. This mechanism also plays a role in aortic constriction (Fig. 5).

Our records showed maximum AVOA correlated well with stroke volume during volume loading (Fig. 3). However, during a brief inflow occlusion, aortic flow was decreased, left ventricular pressure decreased, and maximum AVOA also decreased (Fig. 4). These data suggest that maximum AVOA is correlated well with stroke volume without aortic 

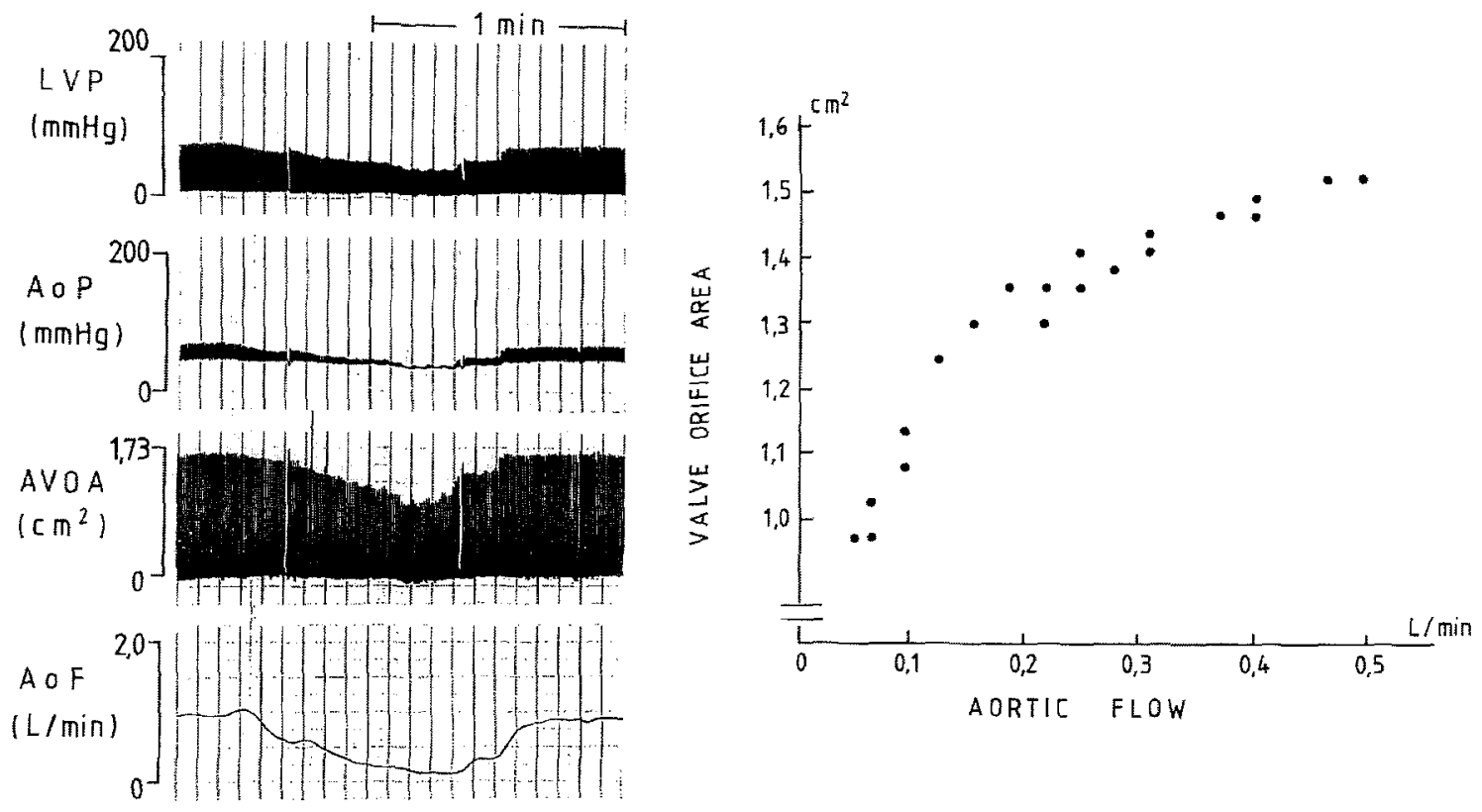

Fig. 4. Left panel, Effects of venous inflow occlusion on AVOA. During a brief inflow occlusion, aortic blood flow $(A O F)$ was decreased, left ventricular pressure $(L V P)$ was decreased, AoP was decreased, and maximum AVOA was decreased. Right panel, Relationship between maximum AVOA and AoF at inflow occlusion-a graph of the AOF and maximum AVOA showed a nonlinear correlation.

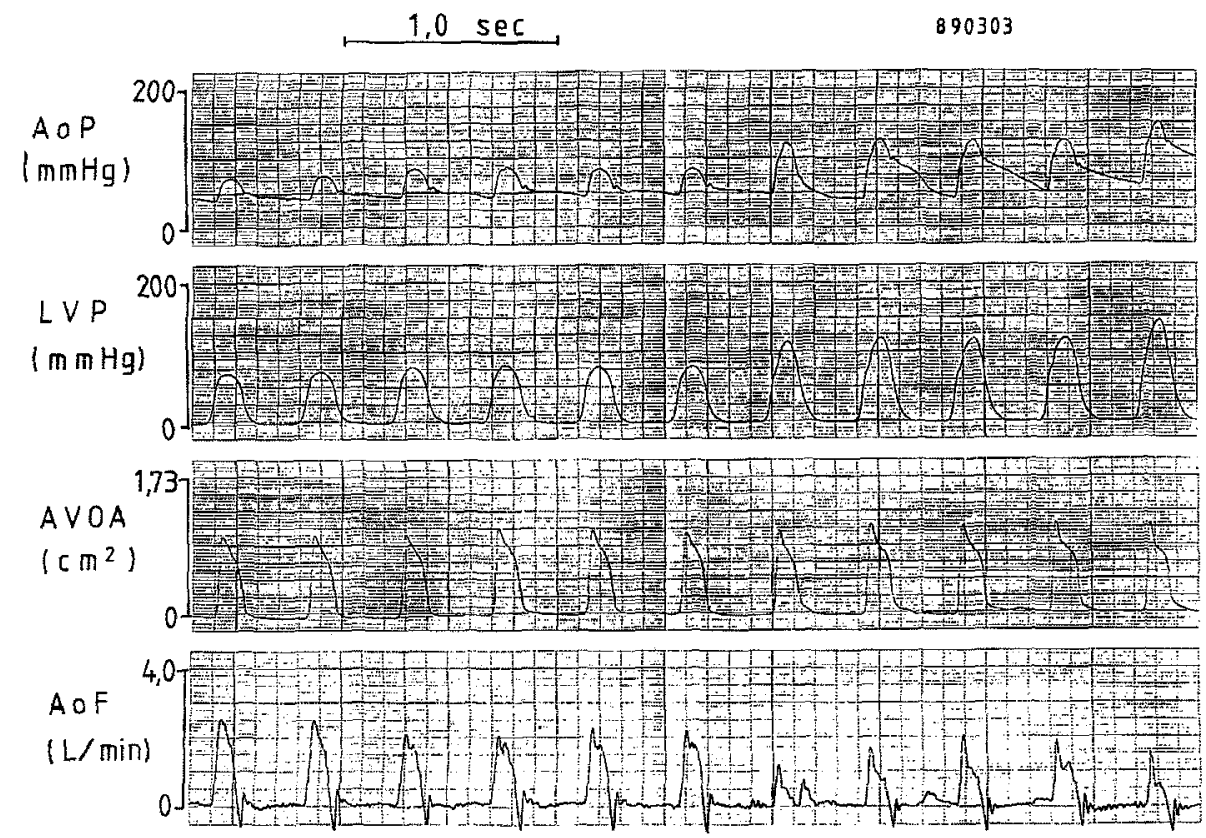

Fig. 5. Effects of constriction of the ascending aorta on phasic changes of (AVOA) area. Although the stroke volume was decreased, maximum AVOA was not decreased. AoP and left ventricular pressure $(L V P)$ were elevated with increase of aortic stenosis. $A o F$, Aortic blood flow. 
constriction. Laniado et al..$^{5}$ also reported that the echographically determined AVOA correlated well with aortic stroke volume $(r=0.94)$. Conversely, during ascending aorta constriction with snares just above the aortotomy, maximum AVOA was not decreased while aortic flow decreased (Fig. 5). This phenomenon suggests that maximum AVOA is mainly affected by aortic pressure in this condition. This difference is due to aortic rooot expansion caused by aortic pressure elevation. Our findings do not entirely contradict Laniado's finding that the maximum AVOA is reflection of aortic flow and aortic stroke volume without aortic stenosis; the aortic pressures were not monitored in Laniado's study. ${ }^{5}$

In their study with an aortic valve model, Bellhouse and Bellhouse ${ }^{1}$ found that the valve opened rapidly before peak flow, stayed open until after peak flow, and moved toward closure as flow started to decelerate. The opening-area curve plateaued during the period of valve opening. They postulated a vortex mechanism which formed in the sinuses and contributed to efficient valve closure. The results presented here differ from those in the literature in two points: opening-area curve and temporal relations. Our results show that AVOA curve is composed of three phases: rapid opening (C1-S1 in Fig. 1), initial slow decreasing (S1-S2 in Fig. 1), and late fast decreasing (S2-C2 in Fig. 1). There was no plateau phase such as Bellhouse and Bellhouse ${ }^{1}$ reported. The reason for this lack of plateau may be the presence of aortic root nonexpansile leaflet attachment and the absence of elastic valve leaflet and full circular opening in their model study.

A surgical implication of this observation is that slicing operation for rheumatic aortic valve lesions is difficult to repair completely without regurgitation because these valve leaflets have not elasticity. Moreover, surgeons should pay attention to avoid reducing commissural expansion at aortic valve repair. The development of bioprostheses with expansile stents or the implantation of stentless valves is to be encouraged.

In conclusion, the importance of cusp expansion and contraction for aortic valve opening and closing is not fully explained in the present study. However, dynamic recordings of AVOA show several findings: (1) the aortic valve already opened before aortic pressure elevation and aortic flow onset, (2) maximum AVOA preceded peak aortic blood flow, (3) initial slow closing was induced by cusp contraction from full round expansion to triangular opening, and (4) maximum AVOA was affected by not only aortic flow but also aortic pressure.

\section{REFERENCES}

1. Bellhouse B, Bellhouse F. Fluid mechanics of model normal and stenosed aortic valves. Circ Res 1969;25: 693-704.

2. Bellhouse B, Talbot $\mathbf{L}$. The fluid mechanics of the aortic valve. J Fluid Mech 1969;35:721-35.

3. Stein PD. Roentgenographic method for measurement of the cross-sectional area of the aortic valve. Am Heart J 1971;81:622-34.

4. Bernuth GV, Tsakiris AG, Wood EH. Effects of variations in the strength of left ventricular contraction on aortic valve closure in the dog. Circ Res 1971;28:705-16.

5. Laniado S, Yellin E, Terdiman R, Meytes I, Stadler J. Hemodynamic correlates of the normal aortic valve echogram: a study of sound, flow, and motion. Circulation 1978;54:729-37.

6. Van Steenhoven AA, Verlaan CWJ, Veenstra PC, Reneman RS. In vivo cinematographic analysis of behavior of the aortic valve. Am J Physiol 1981;240: H286-92.

7. Tamiya K, Higashidate M, Kikkawa S. Technique with lock-in amplifier for real-time measurement of tricuspid valve annulus area. Am J Physiol 1986;251:H236-41.

8. Tamiya K, Higashidate M, Kikkawa S. Real-time and simultaneous measurement of tricuspid orifice and tricuspid anulus areas in anesthetized dogs. Circ Res 1989;64:427-36.

9. Higashidate M, Tamiya K, Kurosawa $H$, Takanashi $Y$, Imai Y. Real-time measurement of tricuspid valve annular area for annuloplasty: experimental study. J ThORAC CaRdiovasc SuRg 1988;96:88-91.

10. Higashidate M, Tamiya K, Kurosawa H, Imai Y. Role of the septal leaflet in tricuspid valve closure. J Thorac Cardiovasc Surg 1992;104:1212-7.

11. Thubrikar M, Bosher LP, Nolan SP. The mechanism of opening of the aortic valve. J THORAC CARDIOVASC SURG 1979;77:863-70.

12. Thubrikar M, Harry R, Nolan SP. Normal aortic valve function in dogs. Am J Cardiol 1977;40:563-8.

13. Thubrikar M, Piepgrass WC, Bosher LP, Nolan SP. The elastic modulus of canine aortic valve leaflets in vivo and in vitro. Circ Res 1980;46:792-800.

14. Brewer RJ, Deck JD, Capati B, Nolan SP. The dynamic aortic root: its role in aortic valve function. $\mathrm{J}$ Thorac Cardiovasc Surg 1976;72:413-7. 